\title{
Lewis acid-base adducts studied by the chemical bond overlap model
}

\author{
Ewerton M. Lima (IC)*, Renaldo T. Moura Jr (PQ). \\ ewerton.m.lima@hotmail.com
}

Department of Chemistry and Physics, Center of Agrarian Sciences, Federal University of Paraíba, Campus II - University City, 58397-000 Areia, PB, Brazil

Keywords: Chemical bond, Lewis acid-base, overlap model.

\section{Introduction}

Experimental studies indicate that the strengths of the Lewis acids in the boron trihalide $\mathrm{BX}_{3}$ series increase in the order $\mathrm{BF}_{3}<\mathrm{BCl}_{3}<\mathrm{BBr}_{3}$ with respect to Lewis bases (i.e. $\mathrm{NH}_{3}$ being this trend the opposite of that expected from the electronegativity order $\mathrm{F}$ $>\mathrm{Cl}>\mathrm{Br}$. Indeed, the atomic charges in the $\mathrm{BX}_{3}$ molecules calculated by different partitioning methods have positive values for the boron atom when $\mathrm{X}$ becomes more electronegative [1]. The most accepted explanation is related to the strengths of the $\mathrm{B}-\mathrm{N}$ $\pi$-bonding in $\mathrm{X}_{3} \mathrm{~B}-\mathrm{NH}_{3}$ adducts, which are attributed to the $2 \mathrm{p}(\mathrm{B})-\mathrm{np}(\mathrm{X}) \pi$ overlaps. In this context, this work aims at investigating the $\mathrm{B}-\mathrm{N}(\mathrm{B}-\mathrm{O})$ chemical bonds in $\mathrm{X}_{3} \mathrm{~B}-\mathrm{NR}_{3}$ $\left(\mathrm{X}_{3} \mathrm{~B}-\mathrm{OR}_{2}\right)$ Lewis adducts by calculating the chemical bond overlap properties $(\mathrm{OP})$. This concept was introduced in the context of the ligand field theory [2]. It was recently developed in our group a methodology for calculating chemical bond overlap properties (e.g. overlap polarizabilities and populations) that uses localized molecular orbitals. It is important to emphasize that this class of systems, with Lewis Acid-Base Adducts, are being used for improving the selectivity and sensitivity of graphene based gas sensors [3], being of interest in material science point of view.

\section{Methodology}

Given the localized character of the chemical bond overlap region, is reasonable to use LMO to obtain the properties associated with it. The total electron density can be decomposed, where the overlap population density can be calculated as written in Eq. 1 .

The overlap contributions for the gross population can be obtained using a numerical integration over the population maps described by $\rho_{\mathrm{OP}}(r)$ in Eq. 1 or using an analytical population analysis where the core and overlap populations are decomposed. Thus, the overlap population can be obtained by the Eq. 2. The

$$
\begin{gathered}
\rho_{\mathrm{OP}}(r)=\sum_{i \in A}^{m} \sum_{j \in B}^{m} c_{i} c_{j} \varphi_{i} \varphi_{j} \\
p_{\mathrm{OP}}=4 \sum_{i \in A}^{m} \sum_{j \in B}^{m} c_{i} c_{j} S_{i j}
\end{gathered}
$$

terms $S_{i j}$ are the overlap integrals on basis functions that can be obtained analytically. The terms $c_{i} c_{j}$ are generally called density matrix elements and can be obtained from a localization procedure for the molecular system. The Coulomb repulsion between two overlap electron densities can be calculated by the Coulomb integral:

$$
\mathrm{J}_{\mathrm{OP}}^{\text {intra }}=\sum_{x i} \sum_{y i}^{M 1} \sum_{z i} \sum_{x j} \sum_{y j}^{M 2} \sum_{z j} \rho_{\mathrm{OP}}\left(r_{i}\right) \cdot r_{12}^{-1} \cdot \rho_{\mathrm{OP}}\left(r_{j}\right) \Delta v_{1} \Delta v_{2}
$$

where $\rho_{\mathrm{OP}}\left(r_{i}\right)$ and $\rho_{\mathrm{OP}}\left(r_{j}\right)$ are points in maps (M1 and M2) of overlap densities and $\Delta v$ are the volume elements. The summations run over mesh points in the electronic population density map and can be solved using an adaptive Monte Carlo method, where the space stratification is done in an adaptive fashion in an iterative process. The chemical bond overlap polarizability can be calculated using the follow expression: 


$$
\alpha_{\mathrm{OP}}^{\kappa \lambda \lambda}=-\frac{2}{F_{\kappa}}\left(\sum_{\substack{i \in A \\ i \in B}}^{m} \sum_{\substack{j \in B \\ j \in A}}^{n} c_{i}^{\prime} c_{j}^{\prime}\left\langle\varphi_{i}|\lambda| \varphi_{j}\right\rangle-\sum_{\substack{i \in A \\ i \in B}}^{m} \sum_{\substack{j \in B \\ j \in A}}^{n} c_{i}^{0} c_{j}^{0}\left\langle\varphi_{i}|\lambda| \varphi_{j}\right\rangle\right)^{\prime} \quad \kappa, \lambda=x, y, z
$$

where $c_{i}^{0}$ and $c_{i}^{\prime}$ are the expansion coefficients for the unperturbed and perturbed LMO. These coefficients can be easily obtained from a SCF procedure followed by a localization unitary transformation.

The studied systems were the Lewis adducts $\mathrm{X}_{3} \mathrm{~B}-\mathrm{B}$ (base), with $\mathrm{X}=\mathrm{F}, \mathrm{Cl}, \mathrm{Br}$ and $\mathrm{B}=\mathrm{NR}_{3}, \mathrm{OR}_{2}$ (with variations of $\mathrm{R}=\mathrm{H}, \mathrm{CH}_{3}, \mathrm{Et}$ ). All calculations were performed with the GAMESS and our BOPP programs, using the wB97X-D/aug-cc-pVQZ method and the Pipek-Mezey localization approach.

\section{Results and discussion}

The results show that the overlap polarizability $\left(\alpha_{\mathrm{OP}}\right)$ for the $\mathrm{B}-\mathrm{N}(\mathrm{B}-\mathrm{O})$ bonds increase in the order $\mathrm{F}, \mathrm{Cl}$ and $\mathrm{Br}$, indicating that this property corroborate the literature results [1]. Noteworthy that this trend can be a consequence of the Lone Pair Bond Weakening Effect, which is stronger in $\mathrm{F}_{3} \mathrm{~B}-\mathrm{NH}_{3}$, decreasing the electron density on the overlap region and its polarizability. Fig. 1a shows the results obtained for the B-N bond

Figure 1. Speedups of different BOPP-1.A-B versions.

a)

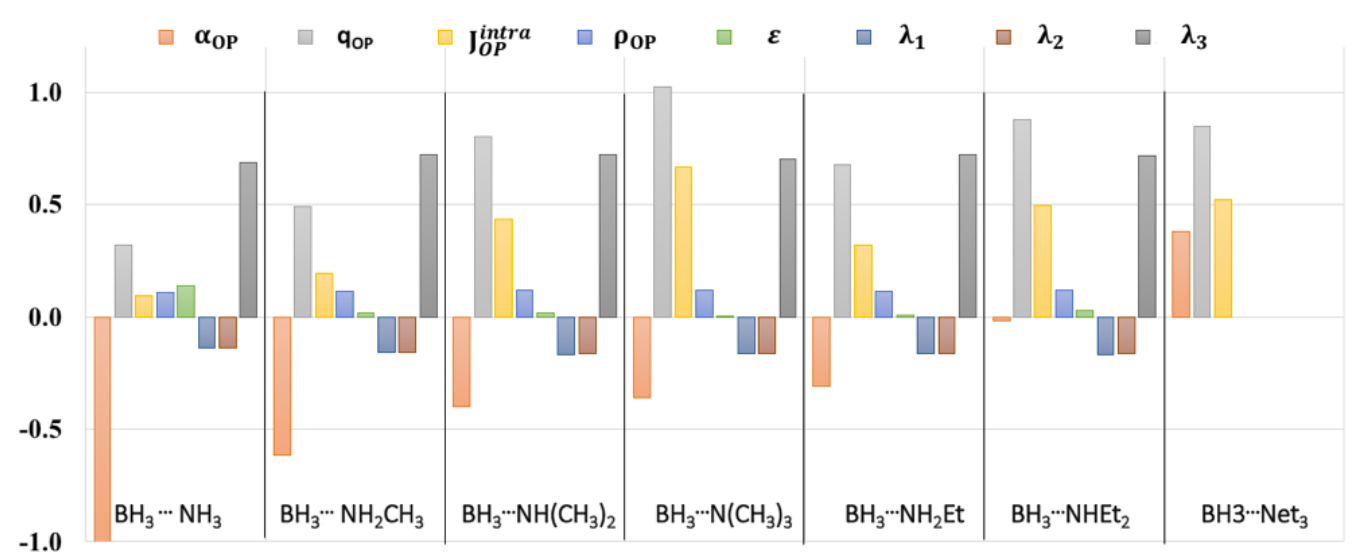

b)
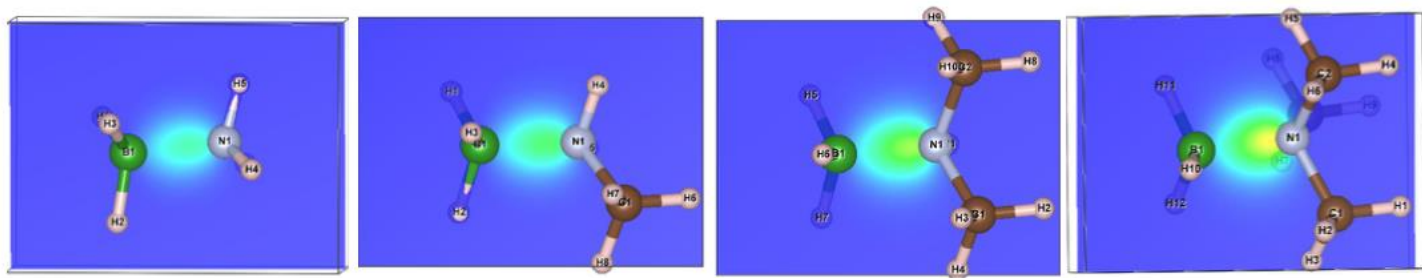

properties in $\mathrm{H}_{3} \mathrm{~B}-\mathrm{NR} \mathrm{NR}_{3}$, including the bond critical points properties. Fig. 1b shows the overlap density increase following the substituent electron donating capability $(\mathrm{H} \rightarrow$ $\mathrm{Me}_{3}$ ). These and other results will be shown at the poster section.

\section{Acknowledgments}

\section{References}

We deeply thank the CNPq, PROPESQ-UFPB and LQTC-UFPE for the support.

[1] F. Bessac, G. Frenking, Inorg. Chem. 42 (2003) 7990

[2] O. L. Malta, H. J. Batista, Chem. Phys. 21 (2002) 282

[3] I. Choudhuri, D. Sadhukhan, P. Garg, A. Mahata, B. Pathak, ACS Sens. 1 (2016) 451 